Cahiers $d u$ MONDE RUSSE

\section{Cahiers du monde russe}

Russie - Empire russe - Union soviétique et États indépendants

$44 / 4 \mid 2003$

Varia

\title{
Semion Lyandres, Dietmar Wulff, eds., A chronicle of the Civil War in Siberia and exile in China
}

\section{Malte Griesse}

\section{OpenEdition}

\section{Journals}

Édition électronique

URL : https://journals.openedition.org/monderusse/4106

DOI : 10.4000/monderusse.4106

ISSN : $1777-5388$

Éditeur

Éditions de l'EHESS

\section{Édition imprimée}

Date de publication : 1 octobre 2003

Pagination : 714-719

ISBN : 2-7132-1833-0

ISSN : $1252-6576$

Référence électronique

Malte Griesse, "Semion Lyandres, Dietmar Wulff, eds., A chronicle of the Civil War in Siberia and exile in China », Cahiers du monde russe [En ligne], 44/4 | 2003, mis en ligne le 06 juillet 2009, consulté le 02 septembre 2022. URL : http://journals.openedition.org/monderusse/4106 ; DOI : https://doi.org/ 10.4000/monderusse.4106

Ce document a été généré automatiquement le 2 septembre 2022.

Tous droits réservés 


\title{
Semion Lyandres, Dietmar Wulff, eds., A chronicle of the Civil War in Siberia and exile in China
}

\author{
Malte Griesse
}

\section{RÉFÉRENCE}

Semion LYANDRES, Dietmar WULFF, eds., A chronicle of the Civil War in Siberia and exile in China. The diaries of Petr Vasil'evich Vologodskii, 1918-1925. Stanford, Hoover Institution Press, Stanford University, 2 vol., 456+472 p.

1 Le journal de Petr Vasil'evič Vologodskij (1863-1925) - avocat, défenseur de la cause sibérienne et sympathisant des sociaux-révolutionnaires, ministre du gouvernement provisoire de Sibérie en 1918, puis, après le coup d'État du général Kolčak, premier ministre de son gouvernement, enfin émigré politique en Chine - est d'un intérêt tout particulier, notamment parce qu'il éclaire le fonctionnement, les conflits et les compromis à l'intérieur du mouvement blanc au pouvoir, mais aussi parce qu'il documente la vie quotidienne et les difficultés des émigrés blancs exilés en Chine.

Les deux éditeurs de cette publication, spécialistes de la Russie révolutionnaire Dietmar Wulff, responsable des annotations de la partie russe (sibérienne) du journal (1918-1920), et Semion Lyandres, qui s'est chargé de la partie chinoise (1920-1925) - ont trouvé l'essentiel du document dans les archives de la Hoover Institution et l'ont complété par des archives privées de la famille du diariste (notamment la partie initiale du journal), qui leur ont fourni des renseignements précieux sur des détails de la vie de Vologodskij largement utilisés dans les notes. Le document, qui compte plus de 1200 pages manuscrites, est entièrement publié et annoté en russe. Il est précédé d'une longue introduction (en anglais) qui le replace dans son contexte historique : guerre civile en Sibérie puis émigration russe en Chine. Les éditeurs donnent un aperçu très détaillé de l'état de la recherche pour les deux phases concernées. Sans nier le caractère dictatorial 
du gouvernement de Kolčak, ils montrent la partialité avec laquelle la plupart des historiens l'évaluent en mettant trop l'accent sur l'aspect militaire, laissant entendre que les affaires civiles auraient été complètement soumises au commandement de l'armée. Le journal de Vologodskij montre cependant que Kolčak, malade pendant plus d'un mois après son arrivée au pouvoir et trop occupé par la guerre, s'est peu mêlé des affaires domestiques et que son gouvernement - dirigé par Vologodskij -, sans avoir les mains totalement libres, a pu néanmoins s'occuper de la politique intérieure civile et surtout de la mise en place d'institutions légales auxquelles tenait particulièrement le premier ministre, juriste de formation et démocrate convaincu.

3 Les éditeurs préviennent le lecteur: le journal de Vologodskij n'attirera pas ceux qui sont à la recherche de documents personnels introspectifs, voire intimes. Vologodskij se veut le chroniqueur de son temps, des décisions et des mesures prises par son gouvernement dans une période particulièrement difficile de l'histoire de la Russie. Il reste toujours fidèle à son souci de fournir des matériaux aux historiens à venir, et c'est dans cet esprit qu'il commence son texte : " J'ai décidé de tenir un journal. La révolution russe traverse visiblement une nouvelle phase de son existence, peut-être plus difficile et plus tourmentée que jusqu'alors» (6/6/1918). Bien que, par la force des circonstances, la nature de son récit change lorsqu'il émigre et s'éloigne des sphères du pouvoir, la partie chinoise reste cependant documentaire et se contente de raconter de façon neutre les événements vécus. Néanmoins, précisément parce que l'auteur est un des acteurs politiques principaux et, de ce fait, amené à prendre des décisions importantes, on trouve aussi dans son journal, souvent entre les lignes, des considérations de valeurs, des conflits moraux qui, sans s'étaler, révèlent au lecteur une personne, avec ses fractures et ses incohérences.

4 Sympathisant, mais jamais membre des SR, Vologodskij s'écarte de plus en plus de ce parti dès la première révolution de 1917, en raison notamment de leur scission et de leur incapacité à mener une politique réaliste orientée vers le pouvoir; cette prise de distance a lieu avant l'écriture du journal. Pourtant, on trouve l'expression d'un renoncement partiel à son " régionalisme » sibérien dans son texte. Sa position à la tête du Gouvernement sibérien provisoire (Vremennoe sibirskoe pravitel'stvo) correspondait tout à fait, dans un premier temps, à ses convictions "régionalistes». Ce n'est que lorsqu'il accepte la présidence du Directoire désormais gouvernement central de toute la Russie (créé lors de la conférence d'Oufa), avec pour effet la dissolution du Gouvernement sibérien, qu'il entre en conflit avec ses opinions. D'où ses tergiversations lorsque ses collègues, dont le SR (de droite) Avksent'ev, lui demandent de prêter serment comme premier ministre du Directoire pour mettre en place rapidement l'activité législative du nouvel organe de pouvoir. Dans son journal, il écrit alors: «Moi-même, j’ai du mal à expliquer mon attitude évasive dans cette affaire. Dans une certaine mesure, je craignais de franchir ce pas. J'avais littéralement peur qu'avec mon adhésion au Directoire, le Gouvernement sibérien disparaisse de l'horizon de la vie sibérienne, et je ne sais pourquoi, mais j'ai regretté ces conséquences. Je voulais tout d'abord savoir au juste quelles conditions le Gouvernement sibérien avait posées pour la délégation de son pouvoir au Directoire russe. Visiblement, c'est mon régionalisme sibérien, ma sibérophilie qui s'est exprimée dans ces humeurs-là » (19/10/1918). Le colonel des troupes anglaises Ward, haut représentant des Alliés et toujours bien renseigné sur les affaires russes, voit curieusement en Vologodskij, qu'il soit ou non traitre à l'« idée sibérienne ", la seule vraie personnalité de tout son entourage. Dans sa description d'un banquet donné à l'occasion de l'unification du Gouvernement sibérien avec le Directoire, 
Ward écrit, hésitant entre ironie et reconnaissance: «Je regardais cette foule excitée avec le plus grand intérêt, et à l'exception d'une seule figure - sorte de mélange entre prêtre méthodiste et frère Plymouth - j'étais frappé par l'absence totale de personnalités parmi les gens présents. La personne que j'ai à l'esprit était le social-révolutionnaire Vologodskij, président du Conseil sibérien, qui avait maintenant transféré son amour pour la Sibérie à toute la Russie » (t. 1, p. 176, note 185).

5 Si la déclaration du Comité central des SR le 24 octobre 1918 à Oufa (qui voit dans le Directoire une forme de rempart réactionnaire à combattre sans pitié) suscite un malaise chez Vologodskij (4/11/1918), mais n'ébranle pas sa conviction que le pouvoir nouvellement créé sert la démocratie et l'établissement d'un État de droit, le putsch militaire fomenté par les cercles autour de Kolčak le 18 novembre 1918 provoque en revanche une crise personnelle beaucoup plus profonde. Le pouvoir réel est entre les mains des militaires et il n'a plus rien à voir avec des principes démocratiques. Vologodskij se rend bien compte qu'en restant dans ses fonctions, sa marge de manœuvre, comme celle de son gouvernement, est sensiblement rétrécie et qu'il risque de n'être plus qu'une marionnette aux mains des généraux. Il a le choix : rester ou se retirer. Et il reste, ce qui a fortement irrité de nombreux intellectuels contemporains de la gauche libérale, mais aussi les historiens. Cependant, l'affirmation du biographe de Kolčak, I. F. Plotnikov, selon laquelle Vologodskij aurait non seulement eu connaissance du plan de coup d'État, mais qu'il l'aurait approuvé d'avance, semble tout à fait invraisemblable au vu du journal qui montre très bien le choc provoqué par les événements.

6 Deux facteurs jouent apparemment en faveur de sa décision de demeurer à son poste : l'influence de son entourage, notamment le comportement de ses collègues ministres ainsi que le renvoi à l'opinion du peuple. À en croire son journal, Vologodskij est appelé en pleine nuit et informé de l'arrestation de quatre membres du Directoire, dont les deux $\mathrm{SR}$, Avksent'ev et Zenzinov, mais ces renseignements restent flous. Après une réunion organisée sur le champ, au cours de laquelle il soupçonne le général Rozanov d'en savoir plus qu'il n'en dit, Vologodskij convoque une réunion d'urgence du Conseil des ministres. Il y exige tout d'abord l'arrestation de l'ataman Krasil'nikov qui aurait apparemment donné l'ordre d'arrêter les ministres. Mais « à ma grande surprise - écrit Vologodskij - ma proposition suscita les objections les plus vives. Particulièrement virulent était le discours de G. K. Gins ». Tous les ministres votent pour la dictature militaire et affirment qu'il n'y a pas d'autre choix. Homme de compromis comme toujours, Vologodskij, qui avait cherché à négocier des arrangements dans les situations les plus précaires (ainsi dans la politique face à la Douma régionale sibérienne), se retrouve cette fois brusquement isolé. D'une part, il a «l'impression que tout ce coup d'État des officiers s'était déroulé au su, peut-être même sous les ordres de certains membres du Conseil des ministres ", d'autre part il est très surpris par le retournement de ses collaborateurs les plus proches comme Gins. Ce dernier fournit l'argument décisif qui, par la suite, restera dominant dans les réflexions et les reconsidérations morales de Vologodskij. "[Gins] démontra que Krasil'nikov n'avait fait que ce qu'il fallait faire depuis longtemps déjà, que son arrestation ne trouverait pas de sympathie dans la société, car tout le monde était mécontent du Directoire depuis longtemps... » (18/11/1918). Vologodskij, toujours persuadé du caractère démocratique de son gouvernement, est submergé par le sentiment de ne plus être à la hauteur de la situation. Et dix jours plus tard, il avoue que le putsch militaire n'a véritablement pas suscité la moindre compassion pour le Directoire auprès de la population (29/11/1918). Le manque de 
soutien populaire devient un élément constitutif dans sa réévaluation rétrospective du Directoire qui, outre son immobilisme lié à l'absence permanente de certains de ses membres, aurait été "un monarque collectif» (voir l'interview du 12 janvier 1919 publiée dans Zarja, citée dans l'introduction, p. 48-49) - condamnation a posteriori qui justifie sa réconciliation avec le nouveau régime.

7 À la réunion du 18 novembre 1918, Vologodskij cède sous l'effet de son isolement : « Je déclarai qu'il n'y avait peut-être vraiment plus d'autre issue, mais que je devais en tout cas me retirer de mon poste. » Mais, immédiatement, « un tas de protestations amicales s'élevèrent. Toutes les objections allaient dans le sens que je devais faire partie des ministres et rester à la tête du Conseil des ministres pour sanctionner le coup d'État par mon nom et pour assurer au Conseil des ministres la confiance dont il avait joui jusqu'ici sous ma présidence. » Avec une franchise presque cynique, ses collègues démontrent ainsi à Vologodskij qu'il est irremplaçable pour assurer la légitimité de la dictature, c'est-à-dire que le destin de celle-ci dépend de sa décision. D'où l'enjeu moral pour notre diariste: " J'étais vraiment troublé et abattu par tout ce qui s'était passé. Et la question de savoir si j'avais bien fait de rester au sein du Conseil des ministres me rongeait» (18/11/1918). Les considérations éthiques aboutissent à une crise personnelle qui s'exprime une semaine plus tard: «Tous ces jours [...] ma conscience me tourmentait parce que j'avais violé mon serment de fidélité au Gouvernement russe, que je n'avais pas eu le courage de refuser le poste de président du Conseil des ministres - soudoyé par les arguments de mes collègues prétendant que c'était comme ça qu'il fallait agir pour préserver la confiance envers le gouvernement... » (25/11/1918).

8 Il faut dire que, pour Vologodskij, le fait même de la dictature fut allégé par l'estime qu'il portait à Kolčak en tant que personne et par le danger d'un nouveau glissement vers la droite en faveur des cercles de généraux qui se plaignaient de l'indulgence du dictateur envers la gauche. Ces cercles soutenaient ouvertement la restauration de l'empire tsariste, danger qui fut d'ailleurs alimenté par les rumeurs d'un nouveau coup d'État en préparation (18/12/1918). Vologodskij cherche à promouvoir, même auprès des généraux, l'idée d'une sorte de front populaire, d'une forte unité de toutes les couches de la société pour la « création d'une grande Russie » (sous-entendu : pour la lutte contre les bolcheviks), et des conditions de paix qui permettraient « au peuple russe de choisir librement, la conscience pure et de tout son cœur, le pouvoir qui lui est cher...» (19/12/1918). La répression cruelle d'un soulèvement bolchevik à Omsk le 22 décembre, démantelé à l'avance par les services de contre-espionnage, provoque d'énormes tensions dans le gouvernement, où Vologodskij arrive finalement à s'imposer contre Kolčak, qui se plie aux remontrances de son premier ministre et ordonne une enquête sévère contre les responsables de cette justice sommaire (22 et 25/12/1918).

9 Par la suite, Vologodskij reste loyal au nouveau régime et ne quitte plus son poste de premier ministre malgré les problèmes que posent de nombreuses scissions au sein du gouvernement. Dans le souci de réconcilier les fractions, il réserve de plus en plus son jugement, se faisant ainsi reprocher son manque d'action et sa faiblesse. Quand il envisage de se retirer, ce n'est plus parce qu'il s'oppose à la dictature militaire, mais parce qu'il a du mal à gérer les conflits déclarés entre ses collègues ministres, et aussi parce qu'il finit par se sentir incapable de continuer à mener les affaires politiques. L'autorité du «Régent Suprême» (Verhovnyj Pravitel') - Kolčak - lui sert souvent d'unique appui dans les querelles entre ses ministres. Son ambiguïté, oscillant du soutien à la dictature d'une part, au maintien de certaines convictions démocratiques de l'autre, 
apparaît clairement en juin 1918 lorsque le Conseil des ministres fait passer une loi sur l'autonomie locale et l'élargissement des compétences des zemstva et des assemblées des villes. Pour Vologodskij, il s'agit d'un grand événement du Parlement - et il résume en détail le débat dans son entier, par crainte d'une éventuelle destruction des procèsverbaux - événement qui ne serait comparable par son importance qu'avec la dissolution du Directoire (24/6/1919) : c'est dire que le coup d'État n'est plus envisagé comme l'échec de la démocratie, tout au contraire, car il aurait aboli un gouvernement quasi monarchique.

En automne 1919, la situation s'aggrave encore, les bolcheviks avancent et, à en juger par les interlocuteurs de Vologodskij, le mécontentement de la population - suscité par l'arbitraire des représentants locaux, par les exactions et les confiscations au profit de l'armée blanche - se tourne en premier lieu contre le Conseil des ministres, non pas contre Kolčak, ce qui réitère la représentation populaire du bon tsar et de ses mauvais conseillers, vrais coupables de tous les maux. Mais Vologodskij ne recourt pas à ce modèle explicatif qui mettrait en question sa vision d'un peuple majeur et mûr pour la démocratie. Il assume sa responsabilité et, après une nouvelle manifestation d'opposition contre sa présence au sein du gouvernement, le Conseil des ministres, à l'initiative de Vologodskij lui-même, propose à Kolčak de choisir un nouveau gouvernement et un nouveau président (29/10/1919). Le 25 novembre 1919, une semaine après l'évacuation du gouvernement d'Omsk à Irkoutsk, Vologodskij est destitué et remplacé par Pepeljaev. Lassé des querelles de fractions incessantes au sein du gouvernement, il accueille la nouvelle avec soulagement. Le poste de responsable de la Commission chargée de préparer l'élection d'une Assemblée constituante, qu'il occupe ensuite, n'a plus rien à voir avec la réalité politique. Bientôt éclate le soulèvement bolchevik à Irkoutsk et le régime blanc de Kolčak est renversé. En janvier 1920, Vologodskij et un certain nombre de ses collègues s'enfuient, cachés dans un wagon des Alliés japonais, à Harbin en Chine.

11 Comme la Russie qu'il vient de quitter, la Chine est morcelée par la guerre civile et dirigée par les warlords, que le pouvoir central a du mal à contrôler - circonstances qui incitent le diariste à des comparaisons avec la Russie. À Harbin, dans la zone des Chemins de fer chinois orientaux (Kitajskaja vostočnaja železnaja doroga - KVŽD) les émigrés jouissent encore de droits extra-territoriaux, mais qui sont menacés en permanence dans la mesure où les bolcheviks déploient une propagande agressive parmi les ouvriers des chemins de fer. Dès la consolidation du pouvoir soviétique en Russie, le nouveau régime mène des pourparlers avec le gouvernement central autour du statut de la zone d'exploitation (polosa otčuždenija) de la KVŽD. La presse russophone d'orientation bolchevique s'en prend particulièrement aux émigrés politiques, membres du gouvernement blanc de Kolčak. Peur et insécurité se nourrissent en outre des rumeurs sur les répressions contre les représentants du régime renversé qui n'ont pas réussi à s'enfuir, ainsi que sur l'exécution de Kolčak le 7 février 1920 à Irkoutsk - rumeurs répandues par des officiers libérés (à cette fin?). En même temps, on essaie de monter les Chinois contre les émigrés blancs en accusant ces derniers d'être responsables du massacre de 5000 soldats chinois, à Blagoveščensk, en 1900, perpétré par l'armée tsariste $(1 / 3 / 1920)$. Lorsque, au cours d'une grève générale, l'extradition de certains ex-ministres est exigée, Vologodskij se voit contraint de fuir Harbin, et commence alors une odyssée à travers la Chine (Shanghai, Tietsin, Bejing) pour fuir la persécution politique mais aussi pour trouver un travail lui permettant de nourrir sa femme et sa fille. 

nouvelles politiques concernant la Russie soviétique, il note également toutes les rumeurs qui courent dans le milieu des émigrés en Chine, mais, le temps passant, il est contraint d'admettre qu'il a sous-estimé la longévité du régime bolchevik. Ne parlant pas chinois, il se met à apprendre l'anglais, peut-être en vue d'une éventuelle émigration aux États-Unis, lieu d'exil très prisé des émigrés (9/2/1924), ou alors en Europe.

C'est grâce aux réseaux d'émigrés russes en Chine qu'il trouve quelque maigre emploi, comme à Tientsin en 1920 à l'aide de Štejnberg, et de retour à Harbin comme avocat à la KVŽD en 1922 par l'intermédiaire de son ami (et ex-ministre des Affaires étrangères), Gins. Néanmoins, Vologodskij est aussi sensible aux clivages qui traversent la communauté et qui prolongent en général les divergences politiques et les sécessions de la guerre civile. Ainsi, en avril 1920 à Tientsin, il partage la même pension qu'un groupe d'officiers du général Semenov (qui s'était séparé du gouvernement de Kolčak en 1919), cohabitation obligée qui l'embarrasse compte tenu de leurs divergences politiques et des débauches dispendieuses auxquelles se livrent ces officiers. Obligé par convenance de fréquenter ses compatriotes, il craint pour sa réputation auprès des autres émigrés (18/4/1920). À côté de la solidarité et de l'entraide, Vologodskij observe aussi des manifestations de rivalité, notamment lorsqu'il s'agit de décrocher des postes - comme parmi les juristes russes employés à la KVŽD qui regardent avec méfiance l'afflux d'émigrés toujours croissant.

Enfin la reconnaissance formelle de l'Union soviétique par le gouvernement chinois en mai 1924, et le contrat sur la collaboration bilatérale dans l'administration de la KVŽD (confirmé par le pouvoir local en Manchourie en septembre de la même année), aggravent considérablement la situation des émigrés sans citoyenneté soviétique ni chinoise : Vologodskij perd son travail. Encouragé par Gins, il entreprend de préparer son journal en vue de le publier dans Arhiv russkoj revoljucii à Berlin, ce qui devait assurer, au moins à court terme, la survie de sa famille. Mais la publication ne se fait pas et Vologodskij sombre dans le désespoir. Il meurt le 19 octobre 1925 dans un hôpital de la Croix-Rouge.

L'excellente édition de ce journal, précieuse pour tous ceux qui s'intéressent à la guerre civile et notamment au mouvement et à l'émigration blancs, est accompagnée d'un index et de brèves notices biographiques d'environ 700 personnages cités dans le texte, ce qui la rend fort utile aussi comme dictionnaire biographique de l'émigration blanche orientale.

Cahiers du monde russe, $44 / 4 \mid 2003$ 